\title{
ON PSYCHOLOGY IN CONTEXT OF GLOBALIZATION
}

Eva Neu 1, Michael Ch. Michailov 1, Peter Birkenbihl 1, Manfred Holler 2, Dieter G. Weiss 3 1 Institute Umweltmedizin c/o ICSD/IAS e.V. POB 340316, 80100 Muenchen (Int. Council Sci. Develop./Int. Acad. Sci. Berlin-Bratislava-Innsbruck-Muenchen-NewDelhi-Paris-Sofia-Vienna) 2 Univ. Hamburg, Economics (Ex-Dean), Hamburg, Germany

3 Univ. Rostock, Inst. Physiol. (Dir.),Rostock, Germany

INTRODUCTION: Enormous global problems are essentially related to education, ecology, economy, medicine, etc. Psychology is of essential importance for total human health (spiritual, mental, emotional, social-somatic). Globalization needs renewal of psychology by philosophy (scientific theory, ethics, etc.) also by new organizatory models.

CONCEPTION: Discussion about IUPsyS/EFPA/American/European incl. Russian-societies, etc.

\section{Enlargement of Executive Committee of} IUPsyS/EFPA/national organizations by a. election of permanent 3-honorary (meritocratic \& triumvirate principles: moral support, continuity), 3-presidents (fixed term), 3-general secretaries,etc.;

b. interdisciplinary commission to

EFPA/IUPsyS incl. scientists from psychology/philosophy/physiology,etc.; c. scientific-political commission - incl. representatives of international societies: psychology/IUPsyS, philosophy/FISP/IAB physiology/IUPS, medicine/CIOMS,etc.; d. Election of permanent (seniors:continuity) \& fixed-term members (flexibility) to the Executive Committee: (sub-)continental representatives from important countries (Afro-/West-Asia; America; Australia/AsiaChina-India-Japan,etc.; Europe: Austria/ France/Germany/Russia/Spain/Italy,etc.)

2. Implication of interdisciplinary topics to congress-programmes for psychology: philosophy, medicine-theology

(Brahmanism-Yoga/Buddhism incl. ZenShintoism/ Christianism-Mosaism/ Confucianism-Taoism/ MohammedanismSufism/ etc.)
3. Organization of common interdisciplinary congresses, see $1 \mathrm{c}$

4. Creation of an International Academy for Psychology with continental/nationalbranches by network, also of institutes to International Universities (proposal by British Nobel Laureate

B.Russell/G.Mensching): intern. UNOemployees, e.g. intern. professors, possibility for whole life work, etc.

CONCLUSION: Realization of proposals [14] by IUPsyS/EFPA/European,etc. societies could be model for scientific\&organizatory renewal supporting UNO-Agenda21 for better health on global level.

References: Michailov, Neu et al. FISP-2018Beijing (in-press) World-Congr.Philos.; -2013Athens, Abstract-Book (AB):464-5\&503-4\&766. 2008/10-Seoul Proc. DVD/ISBN-13, 195202/338-339/363-365. -2003-Istanbul 279-280. Proc.World-Congr.Philos. EACME-2017Barcelona, Congr.Book ed.

Inst.Bioetica/Univ.Ramon Llull, 73-74,125-126. WPA-2018-Mexico (Psychiatry) (in-press). 2011-Buenos Aires, AB:PO1.200. EPA-2018 Nice, Eur.Psychiatry 48/S1, S623\&567\&662. IUPsyS-2012-Cape-Town, IntJPsychol 47:407; -2008-Berlin, 43/3-4:154,248,615,799; -2004 Beijing, AB:49,587. ICPM-2017-Beijing, AB:ID: 648493,648895,648749,648878; 2011-Seoul, AB

189; -2005-Kobe, J.Psychosom.Res. 58:85-86.

Invitation for co-operation: If you are interested please write to (IUM) c/o ICSD/IAS e.V., POB 340316, 80100 Muenchen, Germany, email eva.neu@hotmail.de or fax 0049-89-284686.
Table

\section{PRESENT SITUATION OF HUMANITY -} MISERIES

Pathological Way of Life

Human Psychic and Somatic Degeneration, esp. Cardio-Vascular, Oncologic, Orthopedic Diseases, Infections, Psychic Disorders, e.g.

Stress, Abuse of Alcohol, Drugs, Narcotics, Nicotine, etc.

\section{Ecological Destruction} Atmo-, Hydro-, Litho-, Biosphere

Physical (e.g. Radioactivity), Chemical Pollution, Non-Reversible Gen Manipulation, Annihilation and/or Degeneration of Microorganisms, Plants, Animals, Humanity

\section{Socio-Political Conflicts}

Over-/Under-Population, Hunger Catastrophes Violence, Religion Conflicts, Permanent (incl. Asymmetric) Wars, Chaotic Migration

DEDICATION for moral-scientific support 2018-1988: Austria: E.Busek, K.Lorenz ${ }^{*}$, Belgium: Ch.Perelman, I.Prigogine*, Rep.China: Y.T.Lee*, FL: Prinz A. \& Fürst H.-A. von Liechtenstein, Egypt: N.Mahfouz ${ }^{*}$, France: H.Ahrweiler, J.Dausset ${ }^{*}$, J.-M.Lehn*, L.Néel* Germany: M.Eigen*, K.v.Klitzing* H.Michel*, E.Neher ${ }^{*}$, W.Scheel, Th. von Uexküll, B.Vogel, C.F. von Weizsäcker, H.Zacher, GB: P.Clark, Sir

A.Hewish*, B.Josephson*, Sir J.Kendrew*, Lord Brix A.Hewish*, B.Josephson ${ }^{*}$, Sir J.Kendrew*, Lord Brix
of Lewis, J.Lowy, Lord A.Todd ${ }^{*}$, Israel: S.Deutsch, R. Krausz, R.Rahamimoff, Italy: C.Rubbia*/Vatican City: Krausz, R.Rahamimoff, Italy: C.Rubbia*/Natican City: R.Pegoraro, R.Pozzo, A.Querrualt, Japan: K.Fukur, Y.lkemi, H.Suematsu, Nigeria/S.-Africa: Bishop D.Tutu*, W.Soyinka*, Norway: D.Föllesdal,

L.R.Langslet, Pakistan: S.Mohammed, A.Salam*, R.Siddiqi, Spain: F.Mayor, S.Ochoa*, Sweden: H.Alfven*, B.Samuelson*, J. von Uexküll, Russia: N.Basov*, V.D.-Danilyan, A.Prokhorov*, A.Sacharov*, K.Sudakov, USA-India: P.H.Anderson*,

S.Chandrasekhar*, S.Cohen*, J.Deisenhofer*,

G.Govil, H.B.Khorana* L

G.V.Srivastava, S.Ting*, E. Wiesel*, Sri Yoshi

Prol Laureates
Table

International University - INT. FAC. FOR ANTHROPOLOGY

International Institute for Psychology

ICSD proposes the foundation of paradigmatic institutions, esp. International Universities(IU), Hospitals, Global Ecological Agency, etc. A start of these projects could be the foundation of a first Int. Fac. for Anthropology c/o IU. This faculty should include international institutes for general education in medicine-philosophy, psychology, etc. as well as for special education - physiology, morphology, genetics, pathology, etc. Foundation of international institutes via a network of national institutes, based on new paradigmatic models (administrative and scientific), can promote:

(1) International educational programmes for post graduates in medical philosophy, psychology, physiology, etc. as well as traditional Chinese, Indian, European medicine -psychology, incl. also

(2) Philosophical fundamentals of psychology (ethics, scientific theory, etc.) (3) Interdisciplinary research (incl. physiology, morphology, genetics, psychology, neurology, etc.)

(4) New kind of administration, e.g. 2-3 permanent honorary directors (moral, control function) as well as elected (fixed term) directors, gen. secr., etc.

(5) Possibility for whole life work, etc.

According to epistemology, i.e. scientific theory the medical philosophy and psychology are necessary for an Int. Fac. for Anthropology: An Int. Inst. for Psychology could be a paradigm for education and research in this field conc. multidimensional and scientific approaches. 